\title{
PERCEPÇÃO DO IDOSO INSTITUCIONALIZADO SOBRE O PROCESSO DO ENVELHECIMENTO: UM ESTUDO NO MUNICÍPIO DE GURUPI-TO
}

\section{Perception of the institucionalized older on the aging process: a study in the municipality of Gurupi-TO}

\author{
Érica Sousa Martins - Universidade de Gurupi/ Brasil \\ Regiane Sabino Vieira - Universidade de Gurupi/Brasil \\ Ana Caroline de Andrade - Universidade de Gurupi/ Brasil \\ Lucas Gomes Barcelos - Universidade de Gurupi/ Brasil \\ Jeann Bruno Ferreira da Silva- Universidade de Gurupi/ Brasil
}

\begin{abstract}
RESUMO: O objetivo deste artigo é compreender como os idosos institucionalizados na Casa do Idoso de Gurupi - TO vivenciam as perdas naturais decorrentes do processo de envelhecimento. Método: a pesquisa foi realizada durante período aproximado de 02 meses, totalizando 05 encontros, um com cada idoso. Trata-se de um estudo descritivo e exploratório, com abordagem qualitativa. Os critérios de inclusão foram: institucionalização na Casa do Idoso de Gurupi - TO no período mínimo de seis meses; compreensão do que ouve e capacidade de verbalizar; assinatura do Termo de Consentimento Livre e Esclarecido - TCLE; e capacidade de conclusão da entrevista. Excluíram-se do estudo os idosos que não estiveram em conformidade com os critérios de inclusão. Resultados: Compreendeu-se que os idosos da instituição, vivenciando o luto diante dos declínios físicos, cognitivos e sociais, reconhecem a importância dos cuidados oferecidos pela equipe multiprofissional local, tais como auxílio na alimentação e administração de medicamentos. Porém, notam a falta da assistência familiar, desconhecem a sua medicação, sentem sofrimento físico, psíquico e sentimentos de invalidez. Conclusão: Esta pesquisa possibilitou compreensão sobre a percepção do idoso acerca das perdas naturais decorrentes do processo do envelhecimento. Sugere-se pesquisas sobre o processo do envelhecimento afim de contribuir para realização de ações voltadas à promoção do envelhecimento ativo.
\end{abstract}

Palavras-chave: Envelhecimento. Idosos. Institucionalização. Luto.

ABSTRACT: To comprehend how the institutionalized elderly in House of the Elderly of Gurupi - TO experience the natural losses due to the aging process. Method: the research was conducted during the period of 02 months, totalizing 05 meetings, one with each elderly person. It consists in a descriptive and exploratory study, with qualitative approach. The inclusion criteria were: institutionalization in the House of the Elderly of Gurupi - TO in the minimum period of 06 months; comprehension of what they hear and the ability to verbalize; signing of the Written Informed Consent Form - WICF; and capability to finish the interview. The elderly who was not in conformity with the inclusion criteria were excluded. Results: It was known that the elderly of the institution, experiencing grief facing physical, cognitive and social declines, recognize the importance of the healthcare offered by the local multi professional team, such as feeding help and medicine administration. However, they miss the family assistance, do not know about their medications, experience physical and psychic suffering and invalidation feelings. Conclusion: This research made possible the comprehension of the elderly 
perception about the natural losses arriving with the aging process. It is suggested that more research about the aging process should be made aiming to contribute to new actions promoting the active aging.

Keywords: Aging process. Institutionalization. Grief. Elderly.

\section{INTRODUÇÃO}

Este estudo surgiu a partir de pesquisa realizada na Casa do Idoso no município de Gurupi, Tocantins, no intuito de compreender os sentimentos e vivências sobre o processo do envelhecimento. Cocentino e Viana (2011) enfatizam no processo de envelhecimento do ser humano a vivência das perdas relacionadas à saúde nos aspectos físicos, psicológicos, cognitivos e afetivos quando se refere à separação pela morte de parentes, amigos e companheiros. Na terceira idade, ocorrem de forma natural determinadas doenças, como a demência, pneumonia, neoplasias, cardiopatias, nefropatias, etc. Porém, devido ao avanço da ciência e da tecnologia, a longevidade tem se expandido no mundo, transparecendo a necessidade de proporcionar mais assistência e intervenção de qualidade para a pessoa idosa (CRIPPA et al. 2015).

Conforme exposto, devido ao aumento de idosos na atualidade, torna-se necessário que as instituições de acolhimento e saúde possibilitem atendimento afim de promover o envelhecimento ativo e saudável. Fratezi e Gutierrez (2011) enfatizam a relevância do envolvimento de familiares no processo de institucionalização para que se promova atenção integral ao paciente idoso. Além disso, considera-se que o processo de institucionalização pode ser visto como uma via de mão dupla. Por um lado, o idoso pode aderir aos benefícios à medida que recebe cuidados e orientação das equipes. Por outro, existe uma gama de perdas neste local, devido aos idosos não se sentirem totalmente adaptados ao ambiente (BENTES; PEDROSO; MACIEL, 2012).

As perdas vivenciadas pela pessoa idosa, denominadas como estado de luto (ROSS, 2008), representam um processo que acarreta privações tanto pessoais quanto sociais, devido ao estado de velhice estar estigmatizado pelos aspectos de invalidez. Entende-se a palavra perda não exclusivamente em relação à morte, mas a declínios psíquicos, materiais, físicos, entre outros. Também, consideraram-se outros tipos de perdas, como separação entre entes queridos, o desenvolvimento humano, a morte psicológica, amputações, perda de uma casa ou emprego etc. (AZEVEDO et al. 2015). 
Érica Sousa Martins, Regiane Sabino Vieira, Ana Caroline de Andrade, Lucas Gomes Barcelos \& Jeann Bruno Ferreira da Silva

Os declínios orgânicos e cognitivos são naturais da senilidade, sendo importante ressaltar que medidas paliativas são necessárias para minimizar os impactos que estas perdas podem incidir nos aspectos biológicos, psicológicos e sociais da pessoa idosa (OLIVEIRA; LOPES, 2008). Na ausência dos cuidados mínimos necessários, a vivência no ambiente asilar pode levar a pessoa idosa a desenvolver sentimentos negativos, tais como frustração, solidão e abandono. Então, aceitar a instituição asilar como local a se passar os últimos dias de sua vida tornou-se um desafio, tendo em vista que a não aceitação desta condição influencia diretamente na sua qualidade de vida (PORTO; ROECKER; SALVAGIONI, 2013).

Diante do exposto, esta pesquisa surgiu a partir das seguintes questões norteadoras: quais os sentimentos do idoso frente às perdas naturais do processo de envelhecimento? Existem fatores de risco e protetivos ao envelhecimento ativo nas instituições asilares? Qual(is) influência(s) destes fatores no envelhecimento ativos? De que maneira os idosos percebem os cuidados recebidos pela equipe? O objetivo desta pesquisa desdobrou-se em compreender como os idosos institucionalizados na Casa do Idoso de Gurupi-TO vivenciam as perdas naturais decorrentes do processo de envelhecimento.

\section{MATERIAIS E MÉTODO}

A pesquisa foi realizada na Casa do Idoso, localizada no município de Gurupi, Estado do Tocantins, por período aproximado de 02 meses no ano de 2017, totalizando 5 encontros, um com cada idoso. Trata-se de um estudo descritivo e exploratório, com abordagem qualitativa. Para análise e tratamento dos dados foi empregada o método de análise de conteúdo, conforme Bardin (2009).

O caráter descritivo justificou-se pelo fato de o mesmo possibilitar descrever as características de uma população e do fenômeno em estudo, por meio da precisão dos detalhes (DESLAURIERS; KÉRISIT, 2008). Já o caráter exploratório teve por finalidade proporcionar a maior familiaridade com o problema, com vistas a desenvolver, esclarecer e modificar os conceitos e ideias para estudos posteriores. A pesquisa qualitativa foi empregada para aprofundar a compreensão do fenômeno em investigação, com ênfase nos processos vivenciados e nos significados atribuídos pelos sujeitos entrevistados (MORAES; GALIAZZI, 2011). 
Percepção do idoso institucionalizado sobre o processo do envelhecimento: um estudo no município de Gurupi-TO

A instituição de longa permanência abriga atualmente 22 (vinte e dois) idosos. Destes, 5 (cinco) participaram da pesquisa pois, conforme contato prévio com o gestor responsável pela instituição, apenas esses apresentaram funções cognitivas preservadas, estando em condições plenas de estabelecerem comunicação verbal com os entrevistadores.

Os critérios de inclusão foram: institucionalização na Casa do Idoso de Gurupi no período mínimo de seis meses; compreensão do que ouve e capacidade de verbalizar; assinatura do Termo de Consentimento Livre e Esclarecido - TCLE; capacidade de conclusão da entrevista. Excluíram-se do estudo os idosos que não estiveram em conformidade com os critérios de inclusão.

Não houve teste piloto, os dados foram coletados em único momento de entrevista por meio de roteiro de entrevista semiestruturada, ilustrada na Figura I.

Figura I

\section{ROTEIRO DE ENTREVISTA SEMIESTRUTURADA}

Data da entrevista: Hora de início: Término:

Sujeito $\mathrm{n}^{\circ}$ Iniciais: Idade: Sexo: Masc. ( ) Fem. ( )

Escolaridade: Religião

Tempo de institucionalização:

01 - Como o(a) Sr.(a) adentrou a esta instituição?

02 - Qual foi seu sentimento ao entrar para esta instituição?

03 - Para o(a) Sr.(a), o que significa envelhecer?

04 - Ao envelhecer, quais limitações surgiram?

05 - Possui alguma doença física (crônica)? Qual(is)?

06 - Faz uso controlado de algum medicamento?

07 - Como se sente hoje nesta instituição? Por que?

08 - O senhor(a) gosta de receber visitas?

09 - Recebe visita de algum familiar? Com que frequência?

10 - A sua visão sobre morrer é a mesma de quando era mais jovem?

11 - Nesta instituição, o(a) Sr.(a) acredita receber todo o cuidado necessário para sua saúde?

12 - Teria algo a acrescentar sobre a nossa conversa?

Fonte: autoria própria.

Também foi utilizado um caderno de registro diário de campo para anotação de informações fornecidas pelos entrevistados antes e após o momento da entrevista. Para que não houvesse desconfortos relativos à intimidade e privacidade dos idosos, as entrevistas ocorreram na área de convivência da Casa do Idoso de Gurupi-TO. 
Érica Sousa Martins, Regiane Sabino Vieira, Ana Caroline de Andrade, Lucas Gomes Barcelos \& Jeann Bruno Ferreira da Silva

Todas as entrevistas foram gravadas em um aparelho mp4 e transcritas na íntegra para posterior análise e tratamento dos dados, cada entrevista teve duração aproximada de 30 (trinta minutos). Vale ressaltar que as gravações foram descartadas do aparelho após respectiva transcrição para que ninguém, além dos pesquisadores, tivesse acesso às informações. As transcrições foram mantidas sob absoluto sigilo, estando sua guarda sob total responsabilidade dos pesquisadores durante toda a duração da pesquisa.

O estudo ocorreu conforme previsto na resolução 466/2012 do Conselho Nacional de Saúde, foi submetido ao Comitê de Ética em Pesquisa com Seres Humanos da Universidade de Gurupi sob CAAE: 72823417.7.0000.5518 e aprovado conforme parecer $\mathrm{n}^{\circ} 2.313 .281$.

Os dados foram analisados e interpretados segundo a análise de conteúdo de Bardin (2009). A mesma consiste em um conjunto de procedimentos e técnicas que visam extrair sentido dos textos por meio de unidades de análises que podem ser palavras-chave, termos específicos, categorias e/ou temas, de modo a identificar a frequência com que aparecem no texto, possibilitando fazer inferências replicáveis e válidas dos dados. A mesma é constituída pelas seguintes etapas: pré-análise, exploração do material e tratamento dos resultados, inferência e interpretação.

\section{RESULTADOS E DISCUSSÃO}

A unidade de registro (UR) é a unidade de significação a codificar. Pode ser o tema, palavra ou frase, sendo que a frequência com que aparece uma Unidade de Registro denota-lhe importância, as Unidades de Contexto (UC) representam a categoria macro das UR de acordo com respectivos assuntos e temas, as Categorias Finais de Análise representam o tratamento final das UC. Esses aspectos estão relacionados na Tabela I

Tabela 1 - Unidades de Registro, Unidades de Contexto e Categorias Finais de Análise das entrevistas com idosos de uma Instituição de Longa Permanência de Gurupi, Tocantins. 
Percepção do idoso institucionalizado sobre o processo do envelhecimento: um estudo no município de Gurupi-TO

\begin{tabular}{|c|c|c|}
\hline UNIDADES DE REGISTRO & $\begin{array}{l}\text { UNIDADES DE } \\
\text { CONTEXTO }\end{array}$ & $\begin{array}{c}\text { CATEGORIAS } \\
\text { FINAIS DE } \\
\text { ANÁLISE }\end{array}$ \\
\hline Desconhecimento Medicação & & \multirow{4}{*}{$\begin{array}{c}\text { I - Fatores protetivos } \\
\text { do processo de } \\
\text { institucionalização }\end{array}$} \\
\hline Cuidados recebidos pela equipe & CUIDADOS & \\
\hline $\begin{array}{l}\text { Tratamento de Doenças } \\
\text { Atendimento das necessidades básicas }\end{array}$ & PALIATIVOS & \\
\hline $\begin{array}{l}\text { Sentimento de Satisfação } \\
\text { Necessidade de afeto } \\
\text { Contentamento com vistas }\end{array}$ & CONTENTAMENTO & \\
\hline Vulnerabilidade & PROCESSO DO & \multirow{11}{*}{$\begin{array}{c}\text { II - Fatores de risco } \\
\text { do processo de } \\
\text { institucionalização }\end{array}$} \\
\hline Limitações físicas & ENVELHECIMENTO & \\
\hline Sofrimentos físicos & & \\
\hline Sofrimentos psíquicos & & \\
\hline Aceitação do envelhecimento & & \\
\hline Pensamentos imutáveis sobre a morte & \multirow{4}{*}{ PROCESSO DO LUTO } & \\
\hline Perdas de entes queridos & & \\
\hline Perdas de autonomia & & \\
\hline Pensamento conformista sobre a morte & & \\
\hline Rejeição do Trabalho & SENTIMENTO DE & \\
\hline Sentimento de abandono & INVALIDEZ & \\
\hline
\end{tabular}

Fonte: autoria própria

I - Fatores protetivos do processo de institucionalização: refere-se à percepção dos idosos sobre os cuidados paliativos oferecidos pela equipe e à instituição, em geral.

II - Fatores de risco do processo de institucionalização: descreve as principais perdas inerentes ao processo de envelhecimento, o processo de luto e o sentimento de invalidez do idoso.

A partir da elaboração das Categorias Finais de Análise, foi possível construir os resultados, discutidos a seguir.

\subsection{Caracterização sociodemográfica dos idosos da casa do idoso de Gurupi-TO}


Érica Sousa Martins, Regiane Sabino Vieira, Ana Caroline de Andrade, Lucas Gomes Barcelos \& Jeann Bruno Ferreira da Silva

A Tabela 2 apresenta o perfil dos idosos entrevistados. As variáveis fazem referência à idade, sexo, escolaridade, religião e tempo de institucionalização. Para garantir o sigilo e preservar a identidade dos mesmos, foram atribuídos nomes fictícios.

Tabela 2 - Perfil dos Idosos da Casa do Idoso de Gurupi, Tocantins.

\begin{tabular}{|c|c|c|c|c|c|}
\hline Participante & Idade & Sexo & Escolaridade & Religião & $\begin{array}{c}\text { Tempo de } \\
\text { institucionalização }\end{array}$ \\
\hline $\operatorname{Max}$ & 7 & Masculino & $\begin{array}{c}\text { Não foi } \\
\text { alfabetizado }\end{array}$ & Evangélico & 2 anos \\
\hline Gil & 1 & Masculino & $\begin{array}{c}\text { Não foi } \\
\text { alfabetizado }\end{array}$ & Evangélico & 7 anos \\
\hline Sol & 5 & Feminino & Não foi alfabetizada & Católica & 2 anos \\
\hline Lago & 5 & Masculino & $\begin{array}{c}\text { Não foi } \\
\text { alfabetizado }\end{array}$ & Católico & 4 anos \\
\hline Flor & 6 & Feminino & $\begin{array}{c}\text { Não foi } \\
\text { alfabetizado }\end{array}$ & Evangélica & 5 anos \\
\hline
\end{tabular}

Fonte: autoria própria

De acordo com a Tabela 2, a maioria dos idosos entrevistados (80\%) apresentam faixa etária superior a 80 anos e $20 \%$ com idade inferior a 80 anos. Em relação ao sexo dos idosos entrevistados, há prevalência (60\%) do sexo masculino. Quanto ao grau de escolaridade, $100 \%$ declararam que não foram alfabetizados. Em relação ao tempo de institucionalização, $80 \%$ dos participantes estão institucionalizados há menos de 5 anos, e $20 \%$ acima dos 5 anos.

\section{2 percepção do idoso sobre o processo de envelhecimento}

Quanto às principais limitações decorrentes do processo de envelhecimento, os entrevistados Gil e Lago relataram: "Uai, não podemos escolher. Tenho dificuldades pra andar (GIL)”; "Dificuldades para os cuidados necessários, tenho dificuldade para andar. Hérnia de disco na coluna cervical (LAGO).”

A partir dos relatos acima, infere-se que os aspectos relacionados ao declínio físico foram evidenciados. Em estudos sobre o processo de envelhecimento, Papalia e Feldman (2013) apontam alguns declínios físicos comuns nas pessoas idosas, como alterações cerebrais, funcionamento sexual, sistema motor e sensorial. 
Percepção do idoso institucionalizado sobre o processo do envelhecimento: um estudo no município de Gurupi-TO

Segundo Porto, Roecker e Salvagioni (2013) a velhice não pode ser vista apenas como algo negativo, mas que seja encarada como uma etapa marcada por realizações acumuladas no decorrer da vida.

\subsection{Cuidados paliativos recebidos da equipe profissional}

Os cuidados paliativos são considerados como auxiliadores para pacientes e familiares, trabalhando questões que ressaltam sobre lidar com as alterações físicas, psicológicas, espirituais, enfrentamento dos medos e receios e as expectativas. Também, evidenciando o preparo na autodeterminação no processo de morrer e vida tardia, sabendo-se lidar com as perdas, luto e buscando-se atingir o potencial máximo diante das adversidades emergentes (AZEVEDO et al. 2015).

Neste aspecto, os entrevistados Gil, Sol, Lago e Flor responderam que recebem cuidados da equipe local, contudo, a entrevistada Flor afirmou autonomia: "esse negócio de precisar é fogo! Eu faço tudo sozinha aqui no meu quarto" (FLOR).

Entende-se que os cuidados paliativos são de extrema importância para o pleno envelhecimento saudável nas instituições de idosos. Contudo, deve-se levar em consideração que este processo vulnerabiliza o idoso em suas atividades da vida diária e tal fato acentua-se com a solidão, decorrente do abandono pelos familiares (NUNES et al. 2014). Assim, além da dimensão biomédica, os cuidados paliativos também devem contemplar as dimensões psíquica e afetiva (AZEVEDO et al. 2015).

O cuidado oferecido pela equipe da Casa do Idoso de Gurupi também contempla a administração de medicamentos, conforme as falas dos entrevistados Flor, Gil e Sol: "Eu tomo um pequenininho a noite e de manhã, mas eu não sei os nomes deles, a farmácia que dá sabe, só que eu não sei" (FLOR); "Rivotril e AAS" (GIL); "Aqui me dão um remédio mais não sei que remédio é" (SOL).

Diante aos relatos acima, observa-se que os cuidados médicos e de enfermagem necessários são oferecidos pela instituição, porém os entrevistados Flor e Sol desconhecem quais medicamentos fazem uso. Bem mais que oferecer técnicas de cuidados básicos de saúde, os profissionais devem desenvolver atividades que otimizem o envelhecimento saudável em espaços como as Instituições de Longa Permanência para Idosos, visto que, nestas, os sujeitos se apresentam ainda mais fragilizados por trazerem consigo uma vivência por vezes muito dura de reclusão, solidão e abandono dos familiares (NUNES et al. 2014). 
Érica Sousa Martins, Regiane Sabino Vieira, Ana Caroline de Andrade, Lucas Gomes Barcelos \& Jeann Bruno Ferreira da Silva

Contudo, é necessário ser trabalhado os sentimentos demonstrados pelos idosos durante a visita, pois a vulnerabilidade diante da solidão e do abandono dos familiares é inevitável diante das lembranças.

\subsection{Sentimentos verbalizados durante a visita}

Com intuito de compreender a percepção do idoso sobre visitas de amigos e familiares à instituição, todos os entrevistados (100\%), afirmaram que se sentem bem quando às recebe, contudo na fala de Flor e Sol foi evidenciado o sentimento de solidão: "Pois bem, faleceram todos. Todos falecidos. Fiquei só eu. Mãe, pai, irmão todos falecidos aí fiquei só eu" (FLOR); "Recebo visitas, mas eles demoram muito. Meu filho caçula filho é aleijado também, caiu de cara de bicicleta aí machucou o pé e está aleijado" (SOL).

Por ser uma fase marcada por perdas nos aspectos biológicos, psicológicos e sociais, infere-se que há a associação simbólica entre a velhice e a morte, na qual o idoso vivencia experiências de separação de amigos e familiares. As perdas vividas na velhice estão relacionadas à morte real de amigos e companheiros, ao corpo, ao fim das relações de trabalho, ao relacionamento social e familiar. Tais perdas perpassam tanto a dimensão do físico, em sua concretude, como os universos profissional, social e familiar (CONCENTINO; VIANA, 2011).

\subsection{Sentimentos do idoso sobre a morte}

Segundo Marengo, Flávio e Silva (2009) a sociedade evita conversar sobre o luto, contudo, os profissionais da área da saúde devem oferecer um trabalho de re-humanização referente à morte. Assim, principalmente em instituições de longa permanência, os profissionais ali inseridos devem proporcionar a criação de mais espaços e momentos que visem discutir sobre a dor, o sofrimento e o acolhimento humano.

Ao serem entrevistados se percebiam a morte da mesma maneira de quando eram jovens, os participantes apresentaram as seguintes afirmações: “Quando era pequenininha eu tinha muito medo, era medrosa esse negócio de morte, era meninota. Não adianta ter medo quando Jesus nos faz um chamado a gente né” (FLOR); “Toda vida pensei na morte. Estou aqui pra morrer” (MAX); “A mesma coisa, não tem medo da morte nem penso nisso" (LAGO). 
Percepção do idoso institucionalizado sobre o processo do envelhecimento: um estudo no município de Gurupi-TO

De acordo com Kubler-Ross (2008), o luto é caracterizado pela perda, seja ela fisiológica, material ou social e que possui cinco estados ou fases. Na primeira fase ocorre o sentimento de negação, na segunda o sentimento de raiva, na terceira o corre a barganha, na quarta a depressão e na última fase a aceitação. Para a plena elaboração do processo de luto nos idosos entrevistados, é fundamental que a instituição disponha de ambientes e recursos disponíveis para tal finalidade.

Sobre este aspecto, evidenciou-se a necessidade da inserção de um profissional de saúde mental em tal contexto. Este poderá contribuir para que se promova bem-estar e qualidade de vida dos idosos ali inseridos.

\subsection{O processo de institucionalização}

Tendo em vista que as perdas decorrentes do processo do envelhecimento abrangem dimensões biopsicossociais e requer multiplicidade de cuidados, o fato de residir em uma instituição de longa permanência pode restabelecer a vida de um idoso na sua integralidade. Culturalmente esse estabelecimento é rejeitado pelo simbolismo que carrega, mas por outro lado, paulatinamente está se tornando a alternativa para aqueles que se encontram sozinhos quando envelhecem (BESSA; SILVA, 2008).

No intuito de compreender os sentimentos dos entrevistados diante do processo de institucionalização, Flor e Gil afirmaram que foram trazidos ao local por outro sujeito e que se sentem bem ali. "Me trouxeram aqui e eu conheci a casa aqui né? Eu senti muito sabe, aí me deixaram aqui eu fiquei aqui, estou 5 anos aqui. Me sinto bem né. Que eu vou fazer? Fiquei sozinha aqui e estou aqui" (FLOR). A Joana (nome fictício) me trouxe para cá. Foi bom. Acho bom, porque tem almoço, tem janta e a cama sempre está limpinha" (GIL).

Em contrapartida, os entrevistados Max e Lago afirmaram que adentraram por conta própria e que mesmo assim, também se sentem bem no local. "Cheguei para trabalhar aqui em Gurupi e acabei adoecendo e vir para cá, morava na barra do Córrego. Senti triste porque não conseguia mais trabalhar. Me sinto bem, sinto seguro" (MAX). "Entrei por conta própria. Eu não pude ficar sozinho, eu só tive uma filha, ela se casou e teve que ir embora. Foi bom, tem pessoas pra cuidar. Sinto bem, gosto daqui” (LAGO).

Os entrevistados manifestaram sentimentos de afeto e segurança para com a Casa do Idoso de Gurupi. Estes sentimentos estão diretamente relacionados ao tratamento que recebem da equipe local, como cuidados médicos, alimentação e convivência. 
Érica Sousa Martins, Regiane Sabino Vieira, Ana Caroline de Andrade, Lucas Gomes Barcelos \& Jeann Bruno Ferreira da Silva

\section{CONSIDERAÇÕES FINAIS}

Esta pesquisa possibilitou compreensão sobre a percepção do idoso acerca das perdas naturais decorrentes do processo do envelhecimento. A partir da análise dos relatos dos idosos entrevistados, respondeu-se às questões norteadoras iniciais.

Evidenciou-se que os idosos entrevistados reconhecem a importância dos cuidados oferecidos pela equipe multiprofissional local, tais como cuidados com alimentação e administração de medicamentos. Sobre o processo de institucionalização, questões inerentes ao recebimento de visitas de familiares e/ou amigos, inferiu-se que os entrevistados apresentam resistência em aceitarem a ausência dos mesmos. Contudo, se contentam com a atenção recebida da equipe de saúde local.

Assim, na instituição onde se realizou o estudo, evidenciou-se aspectos satisfatórios perante as percepções dos idosos diante do ambiente em que residem. Ao mesmo tempo, notam a falta da assistência familiar, ocasionando sentimentos que refletem na vida dos idosos institucionalizados, como o sofrimento psíquico e sentimentos de invalidez. Relatam desconhecer sua medicação e sentir sofrimento físico, porém, expressam contentamento.

Percebeu-se que a equipe multidisciplinar fornece atendimento atencioso aos idosos nos cuidados paliativos, como no tratamento de doenças. A experiência de realizar esse estudo foi desafiante, porém gratificante. Sugere-se pesquisas sobre o processo do envelhecimento afim de contribuir para realização de ações voltadas à promoção do envelhecimento ativo.

\section{REFERÊNCIAS BIBLIOGRÁFICAS}

AZEVEDO, Daniel et al. Vamos falar de Cuidados Paliativos. Sociedade Brasileira de Geriatria e Gerontologia - SBGG, 2015. Disponível em: < https://sbgg.org.br/wpcontent/uploads/2015/05/vamos-falar-de-cuidados-paliativos-vers--o-online.pdf > . Acesso em: 26 abr. 2019.

BARDIN, Laurence. Análise de conteúdo. Lisboa: Edições, 2009.

BENTES, A. C. O.; PEDROSO, J. S.; MACIEL, C. A. B. O idoso nas instituições de longa permanência: uma revisão bibliográfica. Aletheia, n. 38-39, p. 196-205, 2012. Disponível em: <http://pepsic.bvsalud.org/pdf/aletheia/n38-39/n38-39a16.pdf>. Acesso em: 26 abr. 2019. 
BESSA, M. E. P; SILVA M. J. Motivações para o ingresso dos idosos em instituições de longa permanência e processos adaptativos: um estudo de caso. Texto \& Contexto Enfermagem, v. 17, n. 2, p. 258-65, abr., 2008. Disponível em:

<http://www.scielo.br/pdf/tce/v17n2/06.pdf>. Acesso em: 26 abr. 2019.

COCENTINO, J. M. B.; VIANA, T. C. A velhice e a morte: reflexões sobre o processo de luto. Revista Brasileira de Geriatria e Gerontologia, v. 14, n. 3, p. 591-600, 2011.Disponível em: <http://www.scielo.br/pdf/rbgg/v14n3/v14n3a18.pdf>. Acesso em: 26 abr. 2019.

CRIPPA, A. et al. Aspectos bioéticos nas aplicações sobre cuidados paliativos em idosos: análise crítica. Revista Bioética, v. 23, n. 1, p. 149-60, 2015. Disponível em: <http://www.scielo.br/pdf/bioet/v23n1/1983-8034-bioet-23-1-0149.pdf>. Acesso em: 26 abr. 2019.

DESLAURIERS, J.P; KÉRISIT, M. O delineamento de pesquisa qualitativa. In: A pesquisa qualitativa: enfoques epistemológicos e metodológicos. Petrópolis: Vozes. p. 127-153, 2008.

FRATEZI, Flávia Renata; GUTIERREZ, Beatriz Aparecida Ozello. Cuidador familiar do idoso em cuidados paliativos: o processo de morrer no domicílio. Ciência \& Saúde Coletiva, v. 16, n. 7, p. 3241-3248, 2011. Disponível em: <http://www.scielosp.org/pdf/csc/v16n7/23.pdf>. Acesso em: 26 abr. 2019.

KUPLER-ROSS. E. Sobre a morte e morrer. São Paulo: Martins Fontes, 2008.

MARENGO, M. O.; FLÁVIO, D. A.; SILVA, R. H. A. Terminalidade de vida: bioética e humanização em saúde. Ribeirão Preto: Medicina, 2009, v. 42, n. 3, p. 350-7.

Disponível em:

<http://revista.fmrp.usp.br/2009/vol42n3/REVTerminalidade_de_Vida.pdf>. Acesso em: 26 abr. 2019.

MORAES, Roque; GALIAZZI, Maria do Carmo. Análise textual discursiva. Editora Unijuí, 2011.

NUNES, J. T. et al. Reflexões sobre os cuidados de enfermagem a idosos institucionalizados. São Paulo: Revista Kairós Gerontologia, v. 17, n. 1, p. 355-373, mar./2014. Disponível em:

<revistas.pucsp.br/index.php/kairos/article/download/21390/15657>. Acesso em: 26 abr. 2019.

OLIVEIRA, J. B. A.; LOPES, R. G. C. O processo de luto no idoso pela morte de cônjuge e filho. Psicol. estud, v. 13, n. 2, p. 217-221, 2008. Disponível

em: <http://www.scielo.br/pdf/pe/v13n2/a03v13n2>. Acesso em: 26 abr. 2019.

PAPALIA, D. E.; FELDMAN, R. D. Desenvolvimento humano. Artmed Editora, 2013.

PORTO, A. R.; ROECKER, S.; SALVAGIONI, D. A. J. O envelhecer e a morte: compreendendo os sentimentos de idosos institucionalizados. Revista de Enfermagem da Universidade Federal de Santa Maria, v. 3, n. 1, p. 35-43, jan./abr. 2013. 
Érica Sousa Martins, Regiane Sabino Vieira, Ana Caroline de Andrade, Lucas Gomes Barcelos \& Jeann Bruno Ferreira da Silva

Disponível em: <https://periodicos.ufsm.br/reufsm/article/download/7205/pdf>. Acesso em: Acesso em: 26 abr. 2019.

\section{Credenciais da/os autora/es}

MARTINS, Érica Sousa. Graduada Psicologia pela Universidade de Gurupi. E-mail: ericasml@hotmail.com

VIEIRA, Regiane Sabino. Graduada Psicologia pela Universidade de Gurupi. E-mail: anyasbino2016@gmail.com

ANDRADE, Ana Caroline de. Graduanda em Psicologia pela Universidade de Gurupi. E-mail: anac.deandrade88@gmail.com

BARCELOS, Lucas Gomes. Graduando em Psicologia pela Universidade de Gurupi. Email: lucasgbarcelos0@gmail.com

SILVA, Jeann Bruno Ferreira da. Mestre em Ciências da Saúde pela Universidade Federal do Tocantins - UFT, Especialista em Saúde Mental pela Faculdade de Ciências Sociais Aplicadas de Marabá - FACIMAB, Psicólogo pela Universidade de Gurupi UnirG. E-mail: jbpsicologia@live.com

Endereço para correspondência: Érica Sousa Martins. E-mail: ericasml@ hotmail.com

Como citar este artigo (Formato ABNT): MARTINS, Érica Sousa et al.. Percepção do idoso institucionalizado sobre o processo do envelhecimento: um estudo no município de Gurupi-TO. Educação, Psicologia e Interfaces, v. 3, n.3, p. 218-230, 2019. DOI: https://doi.org/10.37444/issn-2594-5343.v3i3.169

Recebido: 07/08/2019.

Aceito: 20/11/2019. 